\section{AESTHETIC RESULTS THROUGH TISSUE PRESERVATION}

Building on the successful launch of the $3 \mathrm{i}$ T3 Implant, BIOMET $3 \mathrm{i}$ is launching its 3i T3 External Hex Implants.

The 3i T3 External Hex Implant is a contemporary hybrid implant with a multi-surface topography designed to deliver aesthetic results through tissue preservation:

- Coarse Micron Topography:

A resorbable media blasting process using calcium phosphate particles provides ten micron features, which facilitate blood clot retention along the threaded body of the implant

- Fine Micron Topography: A dual acid-etching process provides a 1-3 micron peak-to-peak surface (OSSEOTITE) that supports platelet activation. At the collar region of the implant, this surface is designed to mitigate the risk of peri-implantitis

- Sub-Micron Topography: The option exists for a more complex topography with the Discrete Crystalline Deposition (DCD) of calcium phosphate nanoparticles. This surface treatment has demonstrated increased integration throughout the early healing process, helping to facilitate bone bonding.

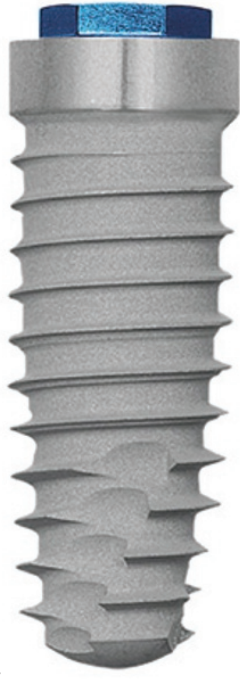

Use of the Gold-Tite Screw with the patented GoldTite surface lubrication allows the screw to rotate further than a non-coated titanium screw. This helps to provide a tight fit between implant components. For more information contact BIOMET 3i on 08006521233.

\section{SMALL FOOTPRINT SCANNER}

3Shape's new D900L 3D scanner brings together RealColor, high speed, implant bar accuracy, and a maximised interior space in a small footprint scanner. D900L scanner features and benefits include:

- Expanded space for larger models - The new D900L scanner allows technicians in labs to scan larger objects including mounted gypsum models, large impression trays, and models with attached articulators

- Designed for speed, accuracy and colour scanning - Scan speed is accelerated by optimised and intelligent 2D image processing algorithms. The unique optical system with $4 \times 5.0 \mathrm{MP}$ cameras ensures high scan speeds, powerful colour capture, enhanced details, and implant bar accuracy

- Save time by skipping the bite scan - With 3Shape's new Auto Occlusion technology, technicians only need to scan upper and lower, and the system automatically calculates occlusion
- Transfer exact articulator positions - Optimised technology now makes it even easier to transfer the exact jaw positions from the physical articulator into the software.

3Shape measures, controls and documents scanner accuracy, validated in accordance with ISO 12836 - a standard which specifies a test protocol for the assessment of crown and bridge accuracy in dental scanners. Implant bars and bridges represent the most accuracy-demanding indication. Complementing the ISO standard, 3Shape additionally validates scan accuracy using specially designed implant objects. www.3shapedental.com

\section{SUPERIOR 3D PRINTING OF DENTAL MODELS}

Stratasys Ltd has announced the immediate availability of VeroGlaze (MED620) dental material for use with its Objet EdenV and OrthoDesk 3D Printers. VeroGlaze enables the 3D printing of dental models with precise A2 teeth colour shade to efficiently produce natural looking dental models with fine details and resolution. The new material can be used in conjunction with all open intra-oral, impression and plaster scanners and is optimised for 3D printing models for crowns, bridge restorations, diagnostic wax-ups, and try-in veneers. VeroGlaze expands Stratasys' growing family of materials for digital dentistry, combining accurate detail visualisation with high dimensional stability. They are used by the Objet EdenV and OrthoDesk line of 3D Printers which print ultrafine 16 micron layers

\section{CUTTING EDGE DIGITAL IMAGING}

For high performance, cutting edge digital imaging, Clark Dental has the solution for you, with a system to meet every need and budget.

For the latest in advanced 3D digital imaging, Clark Dental recommends the Sirona ORTHOPHOS XG 3D. Featuring striking image quality and high image resolution, the XG 3D is suitable for all treatments and can be used in both $2 \mathrm{D}$ and $3 \mathrm{D}$ modes, making it the ideal investment for your practice.

With two scan volumes for dose reduction and time-efficient diagnostics the XG 3D makes digital imaging easy, and with its simple, intuitive controls and automatic patient positioning system, makes for perfect workflow. It is also available with optional CEPH arm on the left or right side.

If you're looking for a new digital imaging system but you're not yet ready to invest in $3 \mathrm{D}$, the $\mathrm{XG}$ is also available as an upgradeable 3D-Ready model. This flexible option means you can evolve with your technology, with the option to step up to 3D when the time is right for you.

For more information call Clark Dental on 01268733

146 or visit the website: www. clarkdental.co.uk. for exceptional detail and finish. www.StratasysDental.com

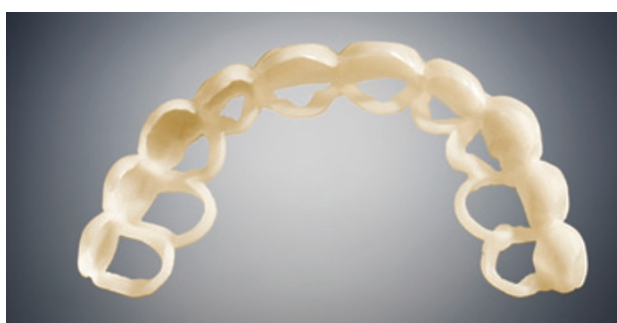

